\title{
Semiclassical Theory of Chaotic Quantum Transport
}

\author{
Klaus Richter ${ }^{(1)}$ and Martin Sieber ${ }^{(2)}$ \\ (1) Institut für Theoretische Physik, Universität Regensburg, 93040 Regensburg, Germany \\ (2) School of Mathematics, University of Bristol, University Walk, Bristol BS8 1TW, England
}

(November 1, 2018)

\begin{abstract}
We present a refined semiclassical approach to the Landauer conductance and Kubo conductivity of clean chaotic mesoscopic systems. We demonstrate for systems with uniformly hyperbolic dynamics that including off-diagonal contributions to double sums over classical paths gives a weak-localization correction in quantitative agreement with results from random matrix theory. We further discuss the magnetic field dependence. This semiclassical treatment accounts for current conservation.

03.65.Sq,73.20.Fz,05.45.Mt
\end{abstract}

Among the prominent wave phenomena which constitute mesoscopic behavior of small phase-coherent conductors, weak localization (WL) represents one key mechanism. This quantum effect shows up as a decrease in the average conductivity with respect to the classical one. WL, originally observed for light and electron waves in disordered samples, has been extensively studied during the last decade for electrons in ballistic conductors, i.e. mesoscopic cavities or quantum dots where the elastic mean free path is considerably larger than the system size. WL is attributed to constructive interference of electron waves which are either coherently backscattered from impurities in disordered systems or multiply reflected at the boundaries of ballistic devices.

In describing ballistic transport semiclassical methods have attracted much interest, since they establish a direct link between quantum transport properties and features of the corresponding classical dynamics, e.g. chaotic, integrable, or mixed behaviour [1,2]. This was demonstrated for clean microstructures in a seminal semiclassical approach [3] to the average reflection in the Landauer framework: the WL peak profile (as a function of a magnetic field) was shown to be Lorentzian for chaotic systems, probing in an impressive way the imprint of the classical dynamics on a measured quantum effect [ 1 . However, while the line shape of the WL peak agreed with results from random matrix theory (RMT), the approach turned out to be inadequate to give the correct WL magnitude for ballistic systems, contrary to the diffusive case [5]. This long-lasting problem to semiclassically obtain the correct leading-order quantum correction to the conductance is related to the so-called diagonal approximation used. It is based on the consideration of a restricted class of pairs of paths built from each backscattered orbit and its time-reversed partner, which moreover violates current conservation [6]. Similarly, WL is not captured in a related semiclassical approach to the Kubo conductivity of ballistic systems [7].

The possible relevance of pairs of non-identical backscattered paths, differing slightly in their initial directions, was first pointed out by Argaman [8]. Later, Aleiner and Larkin [9] approached the problem of ballistic
WL using both, perturbation theory and supersymmetrical methods to derive a RMT result for the conductance. However, their techniques still rely on the presence of quantum scatterers (to regularize the Liouville operator) and strictly speaking do not treat the case of a clean, disorder free, system. Their approach was semiclassically interpreted in Ref. [10] arguing that diffraction-induced small angle scattering is relevant for ballistic WL.

Here we present an adequate, current-conserving semiclassical treatment of the problem to quantitatively describe the average quantum conductance in clean chaotic systems without relying on any diffraction or impurity scattering effects. We consider the leading-order offdiagonal contribution in a semiclassical loop expansion of the Landauer conductance. The relevant off-diagonal terms consist of pairs of orbits which are very close almost everywhere (in configuration space), and differ only in whether they undergo or avoid a self-intersection with small crossing angle 11. . Our results are strictly derived for chaotic systems with uniformly hyperbolic dynamics, but related results for ballistic cavities indicate [14] that they apply to general chaotic systems.

We first compute semiclassical conductance contributions beyond the diagonal approximation in the Landauer framework and later return to the corresponding problem in the Kubo formalism. Consider a two-dimensional, classically chaotic clean cavity with two leads of width $w\left(w^{\prime}\right)$ attached that support $N\left(N^{\prime}\right)$ open channels. The Landauer formula for the conductance $G$ then reads 15 ]

$$
G(E, B)=2 \frac{e^{2}}{h} \sum_{n=1}^{N^{\prime}} \sum_{m=1}^{N}\left|t_{n m}(E, B)\right|^{2} .
$$

Here $t_{n m}(E, B)$ denotes the transition amplitude between incoming and outgoing channels $m$ and $n$ at energy $E$ in the presence of a magnetic field $B$. We first consider the case of time-reversal symmetry, $B=0$, and return to the $B$-dependence of WL later. We assume that the ergodic time is much smaller than the escape time $\tau$ of the cavity and that contributions from direct, lead-connecting processes are negligible. Then the following RMT results for the transmission and reflection amplitudes hold which we give for later reference [16, 17]: 


$$
\begin{aligned}
& \left|t_{n m}\right|^{2}=\frac{1}{N+N^{\prime}+1}=\frac{1}{N+N^{\prime}} \sum_{k=0}^{\infty}\left[\frac{-1}{N+N^{\prime}}\right]^{k}, \\
& \left|r_{n m}\right|^{2}=\frac{1+\delta_{n m}}{N+N^{\prime}+1}=\frac{1+\delta_{n m}}{N+N^{\prime}} \sum_{k=0}^{\infty}\left[\frac{-1}{N+N^{\prime}}\right]^{k} .
\end{aligned}
$$

Our conductance calculation is based on the semiclassical representation of transmission amplitudes [18],

$$
t_{n m} \simeq-\sqrt{\frac{\pi \hbar}{2 w w^{\prime}}} \sum_{\gamma(\bar{n}, \bar{m})} \frac{\Phi_{\gamma} \exp \left[(\mathrm{i} / \hbar) S_{\gamma}\right]}{\left|\cos \theta_{\bar{n}}^{\prime} \cos \theta_{\bar{m}} M_{21}^{\gamma}\right|^{1 / 2}} .
$$

The sum runs over all lead-connecting trajectories $\gamma$ which enter into the cavity at $(x, y)$ with an angle $\sin \Theta_{\bar{m}}=\bar{m} \pi /(k w)$ and exit the cavity at $\left(x^{\prime}, y^{\prime}\right)$ with angle $\sin \Theta_{\bar{n}}=\bar{n} \pi /\left(k w^{\prime}\right)$, where $\bar{n}= \pm n$, and $p=\hbar k$ is the momentum, see Fig. 11(a). In Eq. (价), $S_{\gamma}$ is the classical action, $M_{21}^{\gamma}$ an element of the stability matrix, and $\Phi_{\gamma}=\operatorname{sgn}(\bar{n}) \operatorname{sgn}(\bar{m}) \exp \left[i \pi\left(\bar{m} y / w-\bar{n} y^{\prime} / w^{\prime}-\mu_{\gamma} / 2+1 / 4\right)\right]$ is a phase factor where $\mu_{\gamma}$ contains the Morse index. An expression corresponding to Eq. (4) holds true for $r_{n m}$ in terms of paths reflected back.

The Landauer Eq. (1) contains products $t_{n m} t_{n m}^{*}$ which semiclassically amounts to evaluate double sums over an infinite number of trajectory pairs. In a treatment of the energy-averaged conductance most pairs, consisting of orbits with uncorrelated actions, will cancel each other upon summation. The existing semiclassical approach [3] is based on the diagonal approximation, where only pairs of identical orbits or orbits related to each other by time inversion are taken into account. Then the phase factors from Eq. (4) cancel, and one has $\left|t_{n m}\right|_{\text {diag }}^{2}=\pi \hbar /\left(2 w w^{\prime}\right) \sum_{\gamma(\bar{n}, \bar{m})}\left|\cos \theta_{\bar{n}}^{\prime} \cos \theta_{\bar{m}} M_{21}^{\gamma}\right|^{-1}$.

First we give an alternative further evaluation of this expression employing the sum rule [19,20

$$
\sum_{\gamma\left(y^{\prime}, \theta_{n}^{\prime} ; y, \theta_{m}\right)} \frac{\delta\left(T-T_{\gamma}\right)}{\left|M_{21}^{\gamma}\right|} \simeq \frac{\cos \theta_{n}^{\prime} \cos \theta_{m}}{\Sigma(E)} \mathrm{d} y \mathrm{~d} y^{\prime} \rho(T) .
$$

The sum runs over all orbits with periods $T_{\gamma}$, which begin and end in intervals $\mathrm{d} y^{\prime}$ and $\mathrm{d} y$ around $y^{\prime}$ and $y$ with fixed orientations of the initial and final velocities. $\Sigma(E)$ is the energy surface in phase space; $\Sigma(E)=2 \pi m A$ for billiards of area $A$. The factor $\rho(T) \sim \exp (-T / \tau)$ (for $T \rightarrow \infty$ ) accounts for the exponential loss of particles with velocity $v$ which escape through the openings characterized by the escape rate

$$
\frac{1}{\tau}=\frac{v\left(w+w^{\prime}\right)}{A \pi}=\frac{\hbar}{m A}\left(N+N^{\prime}\right) .
$$

Upon applying the sum rule (5) to the diagonal contribution, integrating over the lead cross sections, and including a factor 4 for each tupel $(\bar{n}, \bar{m})$ one finds for the transmission coefficient of an ergodic system

$$
\left|t_{n m}\right|_{\text {diag }}^{2}=4 \frac{\pi \hbar / 2}{2 \pi m A} \int \mathrm{d} T e^{-T / \tau}=\frac{1}{N+N^{\prime}} .
$$

Correspondingly, the quantum reflection coefficient reads in the diagonal approximation

$$
\left|r_{n m}\right|_{\text {diag }}^{2}=\frac{1}{N+N^{\prime}}+\frac{\delta_{n m}}{N+N^{\prime}} .
$$

The semiclassical evaluation at this level yields the $(k=$ 0 )-term of the RMT result (2.-3). Note that the use of the sum rule (5) allows us to compute individual transmission and reflection coefficients, while Ref. [3] gives results only for the entire classical transmission and reflection.

Summing the first term in Eq. (8) over all channels yields the classical reflection $R=N^{2} /\left(N+N^{\prime}\right)$. The second term in Eq. (8) arises from contributions to $\left|r_{n n}\right|^{2}$ from backscattered orbits paired with their time-reversed partners (elastic enhancement). This gives rise to the diagonal contribution to $\mathrm{WL}, \delta R_{\text {diag }}=N /\left(N+N^{\prime}\right)$, as derived in [3]. In the limit $N=N^{\prime} \rightarrow \infty$ one has $\delta R_{\text {diag }}=$ $1 / 2$, deviating from the RMT result $\delta R_{\mathrm{RMT}}=1 / 4$.
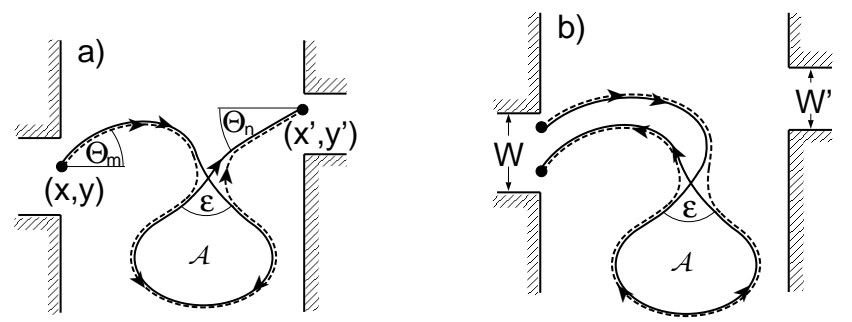

FIG. 1. Sketch of an off-diagonal trajectory pair formed by a self-intersecting classical orbit (solid line) with small crossing angle $\varepsilon$ and a neighboring orbit (dashed) differing mainly in the region around the self-intersection. The paths represent orbits with many reflections at the system boundaries. They contribute to the quantum transmission (a) and reflection (b).

In the following we go beyond the diagonal approximation and consider pairs of different trajectories as sketched in Fig. 11 in coordinate space. They consist of a long, self-intersecting orbit (solid line in Fig. 17a),b)) with small crossing angle $\varepsilon$ forming a closed loop and a second orbit in its close vicinity (dashed line). The two orbits follow the two open trajectory segments, beginning and ending at (exponentially) close points at the lead mouths, in the same but the loop in opposite direction. Given a self-intersecting orbit with small $\varepsilon$ we showed that the neighbouring orbit indeed exists by linearizing the motion in its vicinity 19]. The action difference $\Delta S(\varepsilon)$ of the two orbits is computed by expanding the action around the self-intersecting orbit up to second order. The resulting formula for $\Delta S$, expressed through the elements of the stability matrices for the loop and the two open segments, is rather involved. Hence, we will focus from now on onto chaotic systems with uniformly hyperbolic dynamics characterized by a single Lyapunov exponent $\lambda$ and no conjugate points. One then finds [19]

$$
\Delta S(\varepsilon) \approx \frac{p^{2} \varepsilon^{2}}{2 m \lambda} .
$$


Since a partner orbit is associated with each selfintersection with small crossing angle, we compute the conductance contribution from all such orbit pairs by first summing for each orbit $\gamma(\bar{n}, \bar{m})$ over all $\varepsilon$ selfintersections and finally by integrating over $\varepsilon$. Using Eq. (9) for the action differences occuring in $t_{n m} t_{n m}^{*}$ (with $t_{n m}$ from Eq. (4)) and the sum rule (5) one finds for this 'loop' contribution

$$
\begin{aligned}
\left|t_{n m}\right|_{\text {loop }}^{2} & \simeq \frac{\pi \hbar}{w w^{\prime}} \sum_{\gamma(\bar{n}, \bar{m})} \frac{\delta\left(T-T_{\gamma}\right)}{\left|\cos \theta^{\prime} \bar{n} \cos \theta_{\bar{m}} M_{21}^{\gamma}\right|} I(\varepsilon, T) \\
& \simeq \frac{2 \hbar}{m A} \int \mathrm{d} T e^{-T / \tau} I(\varepsilon, T)
\end{aligned}
$$

with

$$
I(\varepsilon, T)=\operatorname{Re} \int_{0}^{\pi} \mathrm{d} \varepsilon P(\varepsilon, T) \exp \left(\frac{i p^{2} \varepsilon^{2}}{2 \hbar m \lambda}\right) .
$$

In the semiclassical limit $(\hbar \rightarrow 0)$ the contribution from small angles is dominant. In Eq. (11), the density $P(\varepsilon, T)$ of self-crossings with angle $\varepsilon$ for a long orbit of time $T$ can be expressed as an integral over all loops, associated with the self-crossings, with times $T_{\min }(\varepsilon)<t<T$ :

$$
P(\varepsilon, T) \simeq 2 m v^{2} \int_{T_{\min }(\varepsilon)}^{T} \mathrm{~d} t(T-t) \sin (\varepsilon) p_{\text {erg }},
$$

where $p_{\text {erg }}=1 /(2 \pi m A)$ is the ergodic classical return probability. The lower cutoff accounts for the fact that, owing to the exponential divergence of neighboring orbits in a hyperbolic system, a minimum time $T_{\min }(\varepsilon)$ is required to form a closed loop from two trajectories starting at the crossing with initial angular difference $\varepsilon$. Hence $T_{\min }(\varepsilon)$ can be estimated from $c \simeq \varepsilon \exp \left(\lambda T_{\min }(\varepsilon) / 2\right)$ with $c$ of order $\pi$. Detailed numerical and analytical studies 12] have shown that this indeed holds true and that the number of crossings for $T \rightarrow \infty$ is given by

$$
P(\varepsilon, T) \mathrm{d} \varepsilon \sim \frac{T^{2} v^{2}}{\pi A} \frac{\sin \varepsilon}{2}\left[1-2 \frac{T_{\min }(\varepsilon)}{T}\right] \mathrm{d} \varepsilon
$$

with $T_{\min }(\varepsilon)=-(2 / \lambda) \log (\varepsilon / c)$. The integral (11) over the leading-order $T^{2}$ term in Eq. (13) is purely imaginary, and thus its contribution vanishes. However, the contribution to $I(\varepsilon, T)$ of the second, logarithmic term in Eq. (13) is finite and gives - $(\hbar / 2 m A) T$, independent of $\lambda$. We then obtain from Eq. (10)

$$
\left|t_{n m}\right|_{\text {loop }}^{2} \simeq-\left[\frac{\hbar}{m A}\right]^{2} \int \mathrm{d} T T e^{-T / \tau}=\frac{-1}{\left(N+N^{\prime}\right)^{2}} .
$$

Hence, the lack of short loops with $t<T_{\min }(\varepsilon)$ gives rise to a negative quantum correction to the transmission.

Correspondingly, we find for the loop correction to the reflection coefficient

$$
\left|r_{n m}\right|_{\text {loop }}^{2}=-\frac{1+\delta_{n m}}{\left(N+N^{\prime}\right)^{2}} .
$$

Here, as for the diagonal contribution (8), backscattering into the same channel is twice as probable.

Summing over all initial and final channels we obtain for the leading-order quantum transmission and reflection $\delta T_{\text {loop }}=-N N^{\prime} /\left(N+N^{\prime}\right)^{2}$ and $\delta R_{\text {loop }}=-N(N+$ 1) $/\left(N+N^{\prime}\right)^{2}$. For $N, N^{\prime} \gg 1$ we have $\delta R_{\text {diag }}+\delta R_{\text {loop }} \simeq$ $N N^{\prime} /\left(N+N^{\prime}\right)^{2}=-\delta T_{\text {loop }}$. This implies conservation of the average current in the semiclassical limit. Considering off-diagonal terms allows us to semiclassically compute WL corrections consistently either in transmission or reflection. They precisely coincide with the RMT result $\delta T_{\mathrm{RMT}}=-1 / 4$ for $N=N^{\prime} \rightarrow \infty$. Comparison with the RMT results for finite $N, N^{\prime}$ suggests that the $k$ th order terms in Eqs. (2.3) correspond to semiclassical $k$ loop contributions; the diagonal terms are considered as 0 -loop and the orbits in Fig. 11 as 1-loop terms.

Since the closed loops formed by the off-diagonal orbit pairs are traversed in opposite directions, see Fig. 1, these orbits acquire an additional action or phase difference in the presence of a weak magnetic field $B$ due to the flux enclosed. For a uniform perpendicular field the action difference is $4 \pi \mathcal{A} B / \phi_{0}$, where $\mathcal{A}$ is the area of the loops and $\phi_{0}$ the flux quantum. We assume that the distribution $p(t ; \mathcal{A})$ of enclosed areas for trajectories of time $t$ is Gaussian with a system specific parameter $\beta$,

$$
p(t ; \mathcal{A}) \simeq \frac{1}{\sqrt{2 \pi t \beta}} \exp \left(-\frac{\mathcal{A}^{2}}{2 t \beta}\right) .
$$

This is usually well fulfilled for chaotic systems 值邽. For finite $B$-fields we have to perform an additional integration of the field-induced phase differences over the area distribution: $\int_{-\infty}^{\infty} \mathrm{d} \mathcal{A} p(t ; \mathcal{A}) \cos \left(4 \pi \mathcal{A} B / \phi_{0}\right)=$ $\exp \left(-t / t_{B}\right)$, with the magnetic time $t_{B}=\left(4 \pi \beta B / \phi_{0}\right)^{-2}$. Up to timescales $T_{\min }(\varepsilon)$ a negligible flux is enclosed by loops with small crossing angles. We consider this by a respective time shift when inserting $\exp \left(-t / t_{B}\right)$ into the integral (12) over loop lengths:

$$
\begin{aligned}
& P_{B}(\varepsilon, T) \\
& \quad \simeq \frac{v^{2}}{\pi A} \sin \varepsilon \int_{T_{\min }(\varepsilon)}^{T} \mathrm{~d} t(T-t) e^{-\left[t-T_{\min }(\varepsilon)\right] / t_{B}} \\
& \quad \sim \frac{v^{2} t_{B}^{2}}{\pi A} \sin \varepsilon\left[\frac{T}{t_{B}}-1+\frac{T_{\min }(\varepsilon)}{t_{B}}\left(e^{-T / t_{B}}-1\right)+\ldots\right] .
\end{aligned}
$$

In Eq. (17) we used $T_{\min }(\varepsilon) \ll t_{B}$. This corresponds to the original assumption, $T_{\min }(\varepsilon) \ll \tau$, in the range of interest, $\tau \sim t_{B}$. Only the term linear in $T_{\min }(\varepsilon)$ contributes to the integral (11), and we eventually obtain, after computing the $T$-integral (10), a Lorentzian field dependence of the transmission coefficient: $\left|t_{n m}(B)\right|_{\text {loop }}^{2} \simeq$ $\left|t_{n m}(0)\right|_{\text {loop }}^{2} /\left(1+\tau / t_{B}\right)$, A corresponding result applies to $\left|r_{n m}(B)\right|_{\text {loop }}^{2}$. This coincides with the Lorentzian WL lineshape obtained in the diagonal approximation [3], making clear why the diagonal terms already qualitatively account for the WL peak profile. The entire WL 
correction from the diagonal and offdiagonal (1-loop) contribution then reads, in terms of the classical reflection and transmission coefficients $r_{\mathrm{cl}}$ and $t_{\mathrm{cl}}$,

$$
\delta R(B) \simeq \frac{t_{\mathrm{cl}} r_{\mathrm{cl}}}{1+\tau / t_{B}} .
$$

Our refined semiclassical approach to the Landauer conductance yields the correct WL magnitude and lineshape.

The Kubo conductivity reads, in terms of advanced (retarded) Green functions $G^{ \pm}\left(\mathbf{r}, \mathbf{r}^{\prime} ; E\right)$ (for a system of area $A), \sigma=-\left[e^{2} \hbar /(4 \pi A)\right] \operatorname{Tr}\left\{\hat{v}_{x} \Delta G \hat{v}_{x} \Delta G\right\}$, with $\Delta G=G^{+}-G^{-}$. The trace is semiclassically evaluated in position representation by approximating the products of Green functions involved through double sums over classical phase-carrying paths. Pairing identical orbits in the diagonal approximation leads to the classical Kubo conductivity [2]7.8]; off-diagonal terms are again required to compute WL for chaotic systems. As a prototype of an extended clean chaotic system consider, e.g., a twodimensional Lorentz gas. This has been experimentally realized by regular arrays or disordered ensembles of antidots in two-dimensional semiconductor heterostructures [21]. The antidots act as classical scatterers giving rise to diffusive motion on long time scales, while the dynamics for intermediate times is governed by chaotic scattering.

Our semiclassical treatment of WL is based on offdiagonal pairs of paths which have much in common with the orbits discussed above (Fig. 1(b)): they consist of one long self-intersecting trajectory being backscattered after multiple bounces with antidots with nearly opposite momentum and a neighboring orbit which follows the loop formed by the first in opposite direction. A careful treatment of the conductivity trace integral for such pairs of backscattered paths (involving again cutoff times logarithmic in the crossing angle) gives rise to a non-vanishing negative quantum contribution $\delta \sigma$ at $B=0$ [19].

This WL correction for chaotic systems with classical scatterers turns out to coincide with that from disordered systems with quantum impurity scattering. We find

$$
\delta \sigma \simeq-\left(e^{2} / \pi h\right) \ln \left(t_{\phi} / t_{\mathrm{el}}\right),
$$

where $t_{\phi}$ is the phase-coherence time and $t_{\mathrm{el}}$ the elastic scattering time due to reflections at the antidots. Diffusive motion on long time scales, accounted for in a sum rule similar to Eq. (5), is reflected in the ln. Eq. (19) coincides with the result of Ref. [9] for antidot systems when $t_{\phi}$ is large compared to the Ehrenfest time.

The approach above can be generalized to treat linearresponse functions of other observables [19].

To conclude, a semiclassical treatment beyond the diagonal approximation is appropriate to compute quantum corrections to the average conductance in clean chaotic conductors, both in the Landauer and Kubo framework. Chaotic classical dynamics is responsible for a logarithmic angular dependence of the classical return probability, respectively the loops involved, which turns out to be crucial for computing weak localization. Numerical results for billiards [14] show that this $\log \varepsilon$ dependence holds true also for nonuniformly hyperbolic systems indicating that the mechanism presented here is rather general. A semiclassical evaluation of higher-order loop corrections is yet to be performed. While such terms are not negligible for the spectral form factor, the oneloop corrections considered here play the dominant rôle for quantum transport in the mesoscopic regime.

This work was supported by the Deutsche Forschungsgemeinschaft under contract Ri-681/5-1.

[1] For recent reviews see Ref. [2] and R. A. Jalabert, in Proceedings of the International School of Physics "Enrico Fermi" Course CXLIII, edited by G. Casati, I. Guarneri, and U. Smilansky, p. 145 (IOS Press, Amsterdam, 2000).

[2] K. Richter, Semiclassical Theory of Mesoscopic Quantum Systems (Springer, Berlin, 2000).

[3] H. U. Baranger, R. A. Jalabert, and A. D. Stone, Phys. Rev. Lett. 70, 3876 (1993); Chaos 3, 665 (1993).

[4] A. Chang, H. Baranger, L. Pfeiffer, and K. West, Phys. Rev. Lett. 73, 2111 (1994).

[5] S. Chakravarty and A. Schmid, Phys. Rep. 140, 193 (1986).

[6] As a severe shortcoming of the diagonal approximation the magnitude of the WL peak depends on the way it is semiclassically derived and may even vanish 2].

[7] K. Richter, Europhys. Lett. 29, 7 (1995); G. Hackenbroich and F. von Oppen, ibid, 151 (1995).

[8] N. Argaman, Phys. Rev. Lett. 75, 2750 (1995); Phys. Rev. B 53, 7035 (1996).

[9] I. L. Aleiner and A. I. Larkin, Phys. Rev. B 54, 14423 (1996); Chaos, Solitons \& Fractals 8, 1179 (1997).

[10] Y. Takane and K. Nakamura, J. Phys. Soc. Jap. 66, 2977 (1997).

[11] Analogous pairs of periodic orbits have recently been used to derive the $\tau^{2}$-term in the spectral form factor of RMT [12]. These orbit pairs are ballistic analogues of corresponding objects in the diffusive regime 13.

[12] M. Sieber and K. Richter, Physica Scripta T90, 128 (2001); M. Sieber, preprint, 2002.

[13] R. A. Smith, I. V. Lerner, and B. L. Altshuler, Phys. Rev. B 58, 10343 (1998); R. S. Whitney, I. V. Lerner, and R. A. Smith, Waves in Random Media 9, 179 (1999).

[14] M. Turek and K. Richter, unpublished, 2002.

[15] D. Fisher and P. Lee, Phys. Rev. B 23, 6851 (1981).

[16] R. A. Jalabert, J. L. Pichard, and C. W. J. Beenakker, Europhys. Lett. 27, 255 (1994); H. U. Baranger and P. A. Mello, Phys. Rev. Lett. 73, 142 (1995).

[17] C. W. J. Beenakker, Rev. Mod. Phys. 69, 731 (1997).

[18] It is obtained from a semiclassical approximation of the Green functions in the Fisher-Lee relation [15; see [3].

[19] K. Richter and M. Sieber, unpublished.

[20] For a related sum rule see: M. Sieber, J. Phys. A 32, 7679 (1999).

[21] O. Yevtushenko, G. Lütjering, D. Weiss, and K. Richter, Phys. Rev. Lett. 84, 542 (2000). 